\title{
Les enjeux scolaires en France
}

Challenges for France's school system

Los retos escolares en Francia

\section{François Dubet}

\section{CpenEdition}

\section{Journals}

Édition électronique

URL : https://journals.openedition.org/ries/2418

DOI : 10.4000/ries. 2418

ISSN : 2261-4265

\section{Éditeur}

France Education international

Édition imprimée

Date de publication : 1 décembre 2000

Pagination : 53-62

ISSN : 1254-4590

\section{Référence électronique}

François Dubet, «Les enjeux scolaires en France », Revue internationale d'éducation de Sèvres [En ligne], 28 | 2000, mis en ligne le 01 décembre 2003, consulté le 08 juillet 2021. URL : http:// journals.openedition.org/ries/2418; DOI : https://doi.org/10.4000/ries.2418 


\section{Les enjeux scolaires en France}

\section{François Dubet}

\section{Résumé}

En France, l'offre scolaire n'engendre pas l'égalité des chances en raison de l'inégalité sociale des acteurs qui entrent dans une compétition commune. Sans une volonté politique de construction d'un ordre scolaire qui ne soit ni un marché ni une abbaye réservée aux seuls vertueux, l'école ne pourra seule répondre à l'emprise des médias, aux demandes utilitaristes de rentabilité, aux nouvelles technologies qui ont changé la donne.

\section{Challenges for France's school system}

France's school system does not meet its republican goal of ensuring equal opportunity owing to the social inequalities faced by pupils whose abilities are nevertheless judged according to a single standard. Without the necessary political will to build a school system that is neither a free market nor the exclusive reserve of a privileged class, French schools will be unable to rise above the challenges raised by the media's domination, utilitarian demands for cost-efficiency and new technologies which are revolutionising society.

\section{Los retos escolares en Francia}

En Francia, la escuela no ofrece la igualdad para todos a causa de la desigualdad social de los actores que compiten. Sin una voluntad política para elaborar un orden escolar que no sea ni un mercado ni una abadía reservada únicamente a los virtuosos, la escuela no podrá enfrentar sola el poder de los medios de comunicación, las exigencias utilitaristas de rentabilidad, las nuevas tecnologías que lo han cambiado todo. 
Une des rhétoriques familières aux dirigeants est celle de l'urgence : dans l'évolution du monde tel qu'il est, nous n'avons pas véritablement le choix, il faut changer ou disparaître dans une longue décadence. On ne conduit plus les projets de changement en fonction d'objectifs positifs, mais par une réaction de survie face aux épreuves de l'évolution. Dans le débat démocratique, une sorte de darwinisme spontané a remplacé la construction de projets collectifs. Dans ce contexte, il convient alors de dramatiser les débats et les enjeux, de se placer au bord du "gouffre " en hypertrophiant le sentiment de crise, ce qui n'est guère difficile dans les discours sur l'éducation : les élèves ne savent plus rien, l'éducation est réduite à l'animation socioculturelle, la ségrégation scolaire est la règle, les diplômes sont inutiles, la violence est partout, la culture scolaire devient soit inepte, soit le dernier rempart de la civilisation...

Pour définir des enjeux, il n'est pas nécessaire de les dramatiser, mais il faut les construire comme des choix, comme des épreuves de la liberté face à des contraintes objectives dont certaines sont nouvelles, d'autres aussi vieilles que l'école elle-même. On peut distinguer deux niveaux d'enjeux. Le premier concerne les épreuves liées aux mutations lourdes d'un modèle éducatif et scolaire. Le second niveau d'enjeux concerne moins la nature des problèmes que celle des solutions et des types de réponse possibles.

\section{Les mutations d'un modèle}

En France, plus qu'ailleurs, nous sommes attachés, souvent même fascinés ou écrasés, par la référence à une sorte d'âge d'or scolaire, celui d'une école républicaine qui aurait été l'outil de construction de la nation, de la démocratie et du progrès. Parée de toutes les vertus, cette école imaginaire serait emportée dans une longue chute et beaucoup n'imaginent guère d'autre avenir que le retour à un passé dont on ne garde en mémoire qu'une suite de cartes postales. Il faut donc y regarder de plus près.

\section{De l'élitisme républicain à école démocratique de masse : égalité et équité}

Tout système scolaire remplit une fonction de sélection sociale, il distribue des qualifications et hiérarchise des élèves en les armant de manières différentes pour entrer dans la vie. Jusqu'au milieu des années soixante, l'élitisme républicain a articulé de manière très particulière les inégalités sociales et les inégalités scolaires. De façon grossière, on peut considérer que le système scolaire était, dès le départ, clivé entre les grands groupes sociaux: l'école élémentaire était destinée aux enfants du peuple, le lycée aux enfants de la bourgeoisie, alors que les collèges et les établissements professionnels étaient réservés aux classes moyennes et aux catégories supérieures des classes populaires. De façon structurelle, chaque groupe se voyait offrir un type d'enseignement et 
d'espérances scolaires. Dans ce contexte, l'élitisme républicain ne peut pas être confondu avec l'égalité des chances puisqu'il favorise la promotion des meilleurs des enfants du peuple sur la base de leur vertu et de leur excellence, sans les mettre d'emblée dans le même contexte scolaire que les enfants socialement favorisés. Les meilleurs des enfants de paysans seront instituteurs grâce à l'école primaire supérieure, et les meilleurs des enfants d'instituteurs seront professeurs. On sait que ce modèle a offert plusieurs avantages auxquels les plus nostalgiques sont très attachés. Il crée des publics scolaires homogènes, il place l'essentiel de la sélection, "le sale boulot ", en amont de la scolarité, il favorise la promotion des meilleurs sans frustrer les autres puisque les dominants ne sont pas directement menacés par la concurrence des dominés alors que ces derniers n'attendent pas tout de l'école.

Avec la massification scolaire qui s'est poursuivie de façon continue depuis quarante ans, la nature de la sélection a changé, changeant par là-même la nature d'un système devenu école démocratique de masse. Formellement, et ce n'est pas que formel, les élèves sont pris dans le même parcours et sont sélectionnés par l'école elle-même. Même si, à terme, la reproduction sociale se reproduit, la manière dont s'effectue cette reproduction s'est transformée puisque tous les élèves prennent le départ de la même compétition. Tous les élèves sont en concurrence dans la même école et c'est l'école qui opère la sélection de tous les élèves. En France, l'idéal de l'école démocratique de masse a reposé sur un postulat égalitariste selon lequel tous les élèves étant confrontés à la même offre scolaire, ils bénéficieraient des mêmes chances de succès.

On sait que cette politique a donné des résultats contrastés. D'un côté, l'élargissement de l'offre scolaire a permis l'accès de tous à des diplômes de moins en moins rares et, en ce sens, la démocratisation s'est renforcée par l'élargissement de la scolarisation longue à des groupes sociaux qui en étaient exclus. D'un autre côté, les écarts entre les groupes sociaux n'ont pas été sensiblement réduits, ils se sont même accrus aux deux extrémités de la hiérarchie scolaire, dans les grandes écoles et dans les établissements "ghettos». Nous sommes donc confrontés à une déception engendrée par un phénomène structurel. L'égalité de l'offre scolaire, d'ailleurs loin d'être pleinement réalisée, n'engendre pas l'égalité des chances en raison de l'inégalité sociale des acteurs qui entrent dans une compétition commune. De plus, le système qui se massifie se diversifie dans le même mouvement par la multiplication des filières, des types de formations et d'établissements.

Le changement de nature du système scolaire pose un problème politique fondamental qui doit être abordé comme tel. Même s'il est raisonnable d'abandonner l'utopie d'une école annulant complètement les inégalités sociales, il faut au moins que l'école n'accentue pas ces inégalités afin que les élèves gardent confiance en elle. Doit-on imputer cette déception à une absence d'égalité de l'offre scolaire? Dans ce cas, il faut accroître les conditions de cette 
égalité en renforçant l'homogénéité de l'offre scolaire. Au contraire, est-ce l'égalité même de l'offre scolaire qui renforce les inégalités ? Dans ce cas, il faut s'engager résolument dans des politiques de discrimination positive afin d'atténuer les effets des inégalités sociales, il faut choisir l'équité pour atteindre un peu d'égalité.

Il est vraisemblable que les politiques scolaires évolueront entre ces deux pôles : d'un côté elles viseront à accroitre l'égalité, de l'autre elles devront gérer la diversification du système en veillant à ce que les écarts ne se creusent pas trop entre les diverses filières et les diverses formations. Dès lors, cet enjeu se déplacera sur deux thèmes qui sont aussi deux exigences sociales. Le premier est celui de la transparence et de la clarté des règles scolaires afin que les élèves et leurs parents puissent en maîtriser le fonctionnement et y agir de façon efficace. Le second est la multiplication des opportunités de changement d'orientation tout au long de la scolarité. Or, aujourd'hui, notre système est " hypocrite " parce qu'il creuse la distance entre les principes qu'il affiche et la réalité de son fonctionnement, et il est inutilement rigide en enfermant les élèves dans des parcours dont ils ne peuvent guère sortir, comme si toute la vie se jouait avant quinze ans.

\section{La perte du monopole} et l'enjeu de la culture commune

Quand l'école républicaine a été constituée en France, elle s'est d'abord faite contre l'Église, moins par anti-religion ou par anticléricalisme que pour abattre le monopole de l'Église sur ce qu'on pourrait appeler, en reprenant le mot de Durkheim, "la grande culture». À l'exception de la bourgeoisie, de l'aristocratie et de quelques élus, pour la grande majorité de la population, l'Église a longtemps été la seule institution capable d'offrir un accès à une culture universelle, à une culture arrachant les individus aux mondes de leur famille, de leur classe sociale et de leur région. Au nom des Lumières, du progrès et de la Nation, l'école républicaine a progressivement vaincu le monopole de l'Église, non sans s'inspirer d'une forme cléricale. La culture scolaire de l'école élémentaire est devenue le viatique d'une culture citoyenne et la culture du lycée classique s'est imposée comme la cristallisation de la grande culture des humanités et de la science. Pour le dire de façon très simple, l'école est devenue le principal moyen de s'arracher à des appartenances sociales et culturelles étroites, elle a élargi le monde des enfants et des adolescents, elle leur a permis de grandir, elle les a libérés. Bien souvent, l'histoire des boursiers devant tout à l'école est un récit du salut, bien plus que de la promotion sociale. Longtemps aussi, le statut des maîtres et des professeurs a eu quelque chose de sacré parce qu'ils étaient les médiateurs de l'universel de la raison, de la culture et de la science, ce que l'on appelait, en France, la civilisation. 
Qu'on le regrette ou non, depuis trente ans, le monopole de la culture scolaire s'est défait, ce qui n'est pas sans engendrer un sentiment de crise endémique chez les enseignants. Trois grands éléments ont provoqué cette chute. Le premier est l'emprise des médias de masse. La dénonciation rituelle du caractère désordonné, vulgaire et manipulateur des médias n'enlève rien à leur emprise, au fait qu'ils ouvrent au monde tout autant, sinon plus, que la culture scolaire ; pour sortir de leur monde particulier, pour « grandir ", les enfants et les adolescents ont d'autres moyens que la seule culture scolaire, et parfois des moyens terriblement efficaces. Ensuite, il est inévitable qu'une école de masse voie croître les demandes utilitaristes, la recherche de rentabilité des diplômes, puisque les qualifications scolaires sont de plus en plus indispensables pour accéder à un emploi alors que ce n'était pas le cas quand la France comptait plus de $60 \%$ d'ouvriers et de paysans et que la majorité des femmes restaient à la maison. "Gratuite " dans sa vocation, la culture scolaire est de plus en plus soumise à une exigence d'efficacité. Enfin, il est clair que les nouvelles technologies de l'information offrent déjà, et demain plus encore, d'autres modes d'accès aux connaissances.

La culture scolaire - les programmes et les modes d'apprentissage - est donc soumise à une épreuve qui n'oppose pas seulement les anciens aux modernes, mais qui porte sur la définition même de la culture commune. Plusieurs pièges sont clairement identifiables: l'emprise des besoins de formation définis par le marché du travail, l'ouverture sans principe au monde social et à la consommation, le repli conservateur sur les valeurs d'une corporation, la course en avant vers la spécialisation par l'amont... Il est bien évident que la culture scolaire doit viser tout à la fois, elle doit lier à une tradition, ouvrir au monde, préparer à la vie active et développer des mécanismes intellectuels transposables et critiques. Mais ces formules ne sont que des voux pieux et menacent d'empiler les notions et les connaissances si l'on ne parvient pas à trancher sur la nature de la culture commune, celle que devrait posséder le plus mal loti des citoyens.

Il est relativement facile de définir les apprentissages élémentaires, ceux de l'école primaire, et les apprentissages déjà spécialisés, ceux des lycées et de l'enseignement supérieur. Le problème de la culture commune est d'une autre nature car elle englobe des connaissances, des compétences et des dispositions qui ne se réduisent ni aux connaissances élémentaires (lire, écrire, compter), ni aux connaissances indispensables pour faire des études longues et spécialisées qui seront nécessairement différenciées. En fait, cette culture commune, celle du temps du collège, doit être en mesure de répondre au déficit de sens des études que l'on observe aujourd'hui, et de définir ce que doit savoir et ce que doit être un citoyen. Ce choix n'est pas seulement pédagogique, lié aux exigences des études ultérieures, il est aussi politique et il importe que le politique se saisisse d'un problème éminemment politique et ne l'abandonne pas aux seuls profes- 
sionnels de l'éducation. Au moment où les mécanismes de l'intégration sociale sont affaiblis, et où l'école démocratique de masse participe, quoi qu'on en dise, à cet affaiblissement, la capacité de définir la culture commune d'une classe d'âge est un enjeu fondamental.

\section{La place des sujets}

Au cours du siècle qui vient de s'écouler, et là encore que l'on en soit ou non satisfait, la représentation de la socialisation s'est transformée. L'école républicaine, comme l'Église, a été construite comme une institution. Cela signifie que l'humanisme républicain a été conçu comme un travail d'apprentissage de valeurs universelles capables de former un individu socialement intégré, moral, maître de lui-même et susceptible de critique par son adhésion à la Raison et à une culture objective. En France, plus qu'ailleurs, l'instruction et l'éducation ont été distinguées, comme l'étaient l'élève et l'enfant. Bien sûr, il ne faut pas se méprendre, le projet d'instruction était un projet éducatif au sens fort du terme puisqu'il ne s'agissait pas de former seulement des savants, mais aussi des citoyens, des "honnêtes hommes " et des patriotes. Il reste que l'école était largement coupée du monde par l'autonomie de la culture scolaire, la réduction de l'enfant et de l'adolescent au statut d'élève, et un modèle d'autorité pédagogique peu discutable.

Ce système, qui est aussi un modèle, a été fortement déstabilisé. D'abord, avec l'allongement de la scolarité pour tous, les problèmes sociaux sont massivement entrés dans l'école en brouillant la relation pédagogique. Que deviennent la leçon et de la dissertation de philosophie quand elles ne se s'adressent plus à un public composé d'héritiers et de boursiers? Comment " tenir » des adolescents au collège, alors que quarante ans plus tôt ils étaient déjà à cet âge dans la vie active? Ensuite, que cela nous plaise ou non, le XXe siècle a largement accompli le projet individualiste de la modernité. L'enfant et l'adolescent sont considérés comme des sujets capables de créativité et d'autonomie et il est de plus en plus difficile de les tenir aux portes de l'école pour n'y laisser entrer que des élèves. La mixité, les préoccupations professionnelles, les problèmes de chômage et de délinquance, les diverses modes juvéniles ont, de fait, envahi l'école. D'ailleurs on s'en accommode fort bien quand il s'agit des élèves de classes moyennes, beaucoup moins bien quand il s'agit des enfants des classes populaires. L'école a un problème de frontières avec son environnement parce qu'elle n'est plus un sanctuaire.

Dans ce contexte, la socialisation ne peut plus être conçue comme une simple intériorisation de modèles, elle est une construction d'expériences par des individus considérés à la fois comme des sujets moraux et comme des individus utilitaristes. Comme le montre l'évolution de l'école élémentaire, l'enseignement est de plus en plus individualisé et de plus en plus centré sur l'activité de l'élève. L'apprentissage en général, et celui de la citoyenneté en particulier, 
ne peuvent plus reposer sur des modèles d'autorité traditionnels qui déclinent partout ailleurs, notamment dans les familles des classes moyennes dont les enseignants sont le cœur. Nous sommes donc, à l'école comme ailleurs, confrontés à une crise de l'autorité et à la nécessité de fonder un ordre scolaire dans une société démocratique.

Devant cette crise, beaucoup sont tentés d'en appeler aux «bonnes vieilles recettes » du passé laissant accroire que le débat oppose l'ordre à l'anarchie. L'enjeu porte plutôt sur notre capacité, elle aussi politique, de construire un ordre juste. Il ne s'agit pas seulement de définir les frontières séparant les territoires des élèves et ceux des adultes. L'enjeu est bien plus considérable car il faut combiner, afin de les rendre supportables, les deux principes de justice qui commandent la vie scolaire dans une école démocratique de masse. D'un côté, parce qu'elle est démocratique, cette école affirme que tous les élèves sont égaux, de l'autre parce qu'elle est de plus en plus méritocratique, cette école ne cesse de hiérarchiser et de classer les élèves en leur renvoyant la responsabilité de leurs performances. Ceux qui gagnent se sentent confortés, maitres d'euxmêmes et autonomes, ceux qui perdent se sentent humiliés, détruits, incapables... Dès lors, soit ils se détournent de l'école, soit ils deviennent violents et se constituent contre l'école. Le problème qui nous est posé est donc celui de la construction d'un ordre scolaire juste ou perçu comme juste car force est de constater qu'en France comme ailleurs, le développement de l'école de masse a affaibli la croyance dans sa justice, ce qui peut apparaître pour le moins paradoxal. Sans cet effort de construction d'un ordre scolaire acceptable, et qui ne soit ni un marché, ni une abbaye de Thélème réservée aux seuls vertueux, les discours sur les fonctions d'intégration citoyenne de l'école resteront lettres mortes, accroissant ainsi le cynisme ambiant.

\section{Trois enjeux pratiques}

Quels que soient les choix plus ou moins consciemment assumés sur les trois problèmes que nous venons d'évoquer, ils se déclinent en une série de problèmes pratiques qu'il ne sera pas possible d'ignorer longtemps, ou pire, de traiter sans le dire.

\section{Comment réguler le système scolaire?}

Le verbe "réguler " participe d'un jargon technocratique inélégant et cependant précis puisqu'il désigne la formation des règles communes et, surtout, les processus de leur mise en œuvre. Longtemps, l'école républicaine a tiré son homogénéité et sa force de la centralisation du système et de ses capacités d'adaptation silencieuse aux conditions locales. Contrairement à ce qu'on aurait pu imaginer, l'extension et la complexification croissantes de l'école n'ont pas affaibli cette régulation par les règles. Elle s'est même renforcée avec une 
production croissante de textes et de règlements très souvent perdus dans les sables des routines et des conditions locales. Devant cette faible capacité de contrôle effective, le "centre " a produit encore plus de textes et de règlements et le système est devenu encore plus compliqué et encore moins régulé. On sait que derrière des affiches communes, les collèges, les écoles primaires et les lycées ne font pas la même chose, que derrière les ambitions des programmes, chacun se débrouille et que, au bout du compte, l'offre scolaire est de moins en moins homogène. Arrêtée au milieu du gué, l'autonomie des établissements repose sur le dynamisme des équipes pédagogiques et l'intérêt des systèmes politiques locaux. Ce mode de gestion par les règles a aujourd'hui du mal à assurer l'égalité et l'équité.

Quand un système de régulation par les règles est inefficace, une régulation par la demande, c'est-à-dire par le marché, peut apparaître plus efficace. C'est le cas dans un grand nombre de pays, et plus qu'on ne croit en France avec le développement d'un "marché noir " scolaire, mêlant école privée et école publique, et dont seuls les pauvres sont exclus. Ne faudrait-il pas alors étendre aux plus pauvres le droit d'accéder à un marché réservé aux " riches ", notamment aux enfants des enseignants ? Sortir de la carte scolaire, mettre les établissements en concurrence, dénationaliser les examens et garder des concours nationaux. Cette solution ne me paraît pas acceptable car elle accroîtrait les inégalités et l'on peut légitimement considérer que l'éducation n'est pas une marchandise comme une autre. Mais cet appel à la vertu civique ne peut suffire quand on sait que se développe un "marché noir » au sein de l'école publique, et quand on admet que l'école puisse répondre à des contraintes et ne pas les externaliser sous prétexte que les parents seraient égoïstes et les entrepreneurs intéressés. Au nom de quel postulat, les enseignants ne seraient ni l'un ni l'autre?

Entre la régulation par le marché et la régulation par la bureaucratie, il faut développer un mode de pilotage plus politique. Ceci implique que l'autonomie des établissements soit plus grande et que leurs conseils d'administration aient un rôle véritable entre des règles nationales et des demandes sociales que l'école ne peut continuer à ignorer sous prétexte que les élèves, les parents, les élus n'auraient rien à dire en dehors des conditions matérielles de l'éducation. Mais l'accroissement de l'autonomie des acteurs locaux doit être compensée par une régulation centrale plus forte et surtout plus sensible aux résultats obtenus qu'à la conformité des manières de faire. Il ne sert à rien d'énoncer des principes tant que l'on ne se donne pas les moyens d'évaluer leur effectivité et de corriger le tir.

\section{Le métier d'enseignant}

On imagine mal que le métier d'enseignant reste le même ; d'ailleurs il n'a cessé de se transformer, y compris chez les plus attachés aux traditions, puisqu'il faut bien s'adapter aux élèves tels qu'ils sont. Le problème n'est pas de 
savoir si les enseignants seront des "savants" ou des "pédagogues", ils sont déjà et seront toujours les deux, pas plus qu'il n'est de savoir si les enseignants devront apprendre à utiliser d'autres outils de communication et d'information que le livre et le tableau, ils le feront nécessairement parce que les élèves utilisent déjà ces outils vernaculaires... Bref, le métier change, plus vite dans les écoles élémentaires et l'enseignement professionnel que dans les collèges et les lycées, plus vite dans les établissements défavorisés que dans les établissements " chics", mais il change.

Ce changement est contradictoire et peu maîtrisé parce qu'il suit deux lignes opposées. D'un côté, on peut observer une sorte de dilution de l'activité pédagogique dans une succession de tâches éducatives dont on peut craindre que personne ne les contrôle réellement et ne sache à quoi elles servent. D'un autre côté, on assiste à une taylorisation continue du travail éducatif avec la multiplication des intervenants auprès des élèves «à problèmes ", la discipline, les activités spécifiques, l'enseignant se repliant dans sa classe et sur sa discipline défendue comme un pré carré et une affaire d'honneur. Ces deux tendances, au cœur de la formation des professeurs, ne sont pas sans poser de problèmes et là encore, elles appellent des arbitrages sur le statut des enseignants et la définition de leurs tâches. On ne peut en appeler au rôle éducatif de l'école et s'en décharger sur d'autres professions, généralement instables, pas plus qu'on ne peut laisser la transmission des connaissances se dissoudre dans des activités sociales. Il faudra bien trancher sur la définition du service des enseignants si l'on ne veut pas voir se prolonger les dérives provoquées par une pure logique d'adaptation, dérives que tous les « camps " condamnent.

\section{La place de l'école dans l'éducation}

Depuis trente ans, l'école est un univers en extension. En France bien plus que dans d'autres pays, on est tenté d'attendre beaucoup trop de l'école : former des professionnels, assurer l'intégration sociale, former des citoyens et des sujets, réduire les inégalités sociales, attacher à une histoire, définir l'avenir... Longtemps proche d'un ordre régulier, l'institution scolaire se sécularise de manière incontrôlée. Il n'est guère de problèmes sociaux ou éducatifs pour lesquels on n'attende pas de solutions scolaires. La scolarisation de tous a été perçue comme une réponse aux dérives des banlieues, l'allongement indéfini des études s'est imposé comme une solution au chômage des jeunes. L'emprise de l'école est d'autant plus excessive qu'elle ne peut régler tous les problèmes et qu'elle déçoit nécessairement, qu'elle écrase les acteurs, enseignants et élèves, sous le poids d'ambitions parfaitement irréalistes.

Mais en même temps que l'emprise de l'école s'étend, celle-ci garde une rigidité toute monacale. Au moment où l'on admet que les parcours de vie sont moins fortement programmés et prévisibles, où les carrières professionnelles 
seront multiples, où les connaissances changent constamment, où nous vivons dans de multiples cultures, l'école se charge de tous les fardeaux en les pliant à ses propres règles. L'âge des élèves reste un critère essentiel de la carrière scolaire, les choix réalisés à quinze ans sont, en fait, irréversibles, les disciplines ne communiquent guère entre elles, alors qu'elles se mêlent dans la vie professionnelle et dans la recherche... Ainsi se creuse la distance entre le monde scolaire et le monde social. Cette distance reste nécessaire parce que l'école est toujours partiellement hors du monde et ne peut se borner à répondre aux besoins, d'ailleurs contradictoires, de la société. Mais il ne faut pas pour autant que les demandes d'éducation croissantes soient toutes transformées en demandes scolaires. Dans une société qui sera de plus en plus éducative, dans laquelle les demandes de formation ne cesseront de se développer, un des enjeux de demain est la définition des frontières de l'école ; elle doit savoir quel est son domaine.

$$
* *
$$

Tous les enjeux et tous les problèmes que nous venons d'esquisser sont surplombés par un enjeu unique: la capacité politique de se saisir des problèmes scolaires. Or, même dans un pays caractérisé par une école publique puissante et centralisée, cette capacité est étonnamment faible. Depuis vingt ans, tous les ministres qui ont essayé de réformer le système scolaire se sont cassé les dents. De manière routinière, en votant le budget de l'Éducation nationale le Parlement a le sentiment de faire une politique scolaire. En fait, tout se joue entre un ministre, son administration et les syndicats. Ce sont les acteurs qui vivent de l'Éducation nationale qui décident de son destin. Mais autant il est normal que les professionnels de l'éducation aient un rôle dans la définition des politiques éducatives, autant il est choquant qu'ils finissent par en être tenus pour les propriétaires.

Paradoxalement, alors qu'il existe une passion française pour l'école et l'éducation, il existe aussi un silence politique sur ces problèmes, peut-être d'ailleurs en raison de cette passion et des risques qu'elle fait encourir. Pourtant, la capacité de se saisir des enjeux de l'école ne dépend pas des seuls acteurs de l'école. Au contraire même, pour des raisons tenant aux équilibres internes de la machine, aux questions de rang et d'honneur, ces acteurs ne peuvent pas répondre à des enjeux d'une telle ampleur. L'école républicaine est le fruit de la politique, du courage politique arbitrant entre des intérêts sociaux et portant une représentation de la vie sociale. Si nous ne retrouvons pas cette capacité politique, l'école sera engloutie sous son propre poids et sous des querelles qui n'intéresseront plus qu'elle-même. L'institution n'aura plus de fidèles ou de croyants, elle n'aura que des clients dont certains seront salariés, d'autres pas, et ils iront voir ailleurs. 